\title{
Data Monitoring Committee
}

National Cancer Institute

\section{Source}

National Cancer Institute. Data Monitoring Committee. NCI Thesaurus. Code C142489.

A group of experts that periodically assess information from an ong oing clinical study, including participant's safety and trial effectiveness. 